\title{
The distractor frequency effect in a delayed picture-word interference task: further evidence for a late locus of distractor exclusion
}

\author{
Elisah Dhooge • Robert J. Hartsuiker
}

Published online: 10 November 2010

(C) Psychonomic Society, Inc. 2010

\begin{abstract}
A picture-word interference experiment examined the origin of the distractor frequency effect, the effect that pictures are named slower in the context of low-frequency than high-frequency words (Miozzo \& Caramazza, Journal of Experimental Psychology: General, 132, 228-252, 2003). We compared two accounts of the effect: an early, input-related account and a late, response-related account. Participants named high and low-frequency pictures with low and highfrequency distractors in two conditions. In the immediate naming condition, picture and distractor were presented simultaneously. In the delayed naming condition, the distractor was presented 1,000 ms after the picture; pictures had to be named upon distractor presentation. There was a distractor frequency effect in both conditions, but an effect of picture frequency only in the immediate naming condition (showing that in the delayed naming condition, lexical selection had been completed). These results support a late origin of the distractor frequency effect.
\end{abstract}

Keywords Distractor frequency effect · Picture-word interference task - Response exclusion hypothesis .

WEAVER $++\cdot$ Word production

\section{Introduction}

A central issue in language production concerns the selection of words from the mental lexicon: lexical

E. Dhooge $(\triangle) \cdot$ R. J. Hartsuiker

Department of Experimental Psychology, Ghent University,

Henri Dunantlaan 2,

9000 Ghent, Belgium

e-mail: elisah.dhooge@ugent.be

R. J. Hartsuiker

e-mail: robert.hartsuiker@ugent.be selection. There is consensus that a spreading activation mechanism at the semantic level will lead to the activation of semantically related lexical representations (e.g., Levelt, Roelofs, \& Meyer, 1999), but the precise decision mechanism needed to select the correct representation is still under debate. Research on the dynamics of lexical selection often employs the picture-word interference (PWI) paradigm (for an early review, see MacLeod, 1991). In this paradigm, the participant is presented with a picture with a superimposed word (i.e., distractor). The task is to name the picture whilst ignoring the distractor. Within this paradigm, it is possible to vary the relationship between the picture and distractor (e.g., a semantic relationship such as CATdog, e.g., Schriefers, Meyer, \& Levelt, 1990) and the properties of the picture and word individually (e.g., Miozzo \& Caramazza, 2003).

One effect that has recently attracted attention is the distractor frequency effect. In a PWI task, Miozzo and Caramazza (2003, see also Burt, 2002; Catling, Dent, Johnston, \& Balding, 2010; Dhooge \& Hartsuiker, 2010) presented pictures with low-frequency or high-frequency distractors. Pictures presented with a high-frequency distractor were named faster than pictures presented with a low-frequency distractor. Currently, there is debate regarding the exact origin of the distractor frequency effect. Two models can account for the distractor frequency effect: WEAVER ++ which assumes an early locus (e.g., Roelofs, 2003, 2005) and the response-exclusion hypothesis (e.g., Mahon, Costa, Peterson, Vargas, \& Caramazza, 2007), which places the effect at a late, postlexical level. The goal of this article is to contrast these two accounts.

In WEAVER++ (e.g., Roelofs, 2003, 2005), information is retrieved from a network by means of spreading activation through a number of stages, namely conceptual identification, lemma retrieval, word form encoding, and 
articulatory processing. Importantly, WEAVER++ assumes input filtering based on attentional processes. When naming a picture with a superimposed distractor, processing of the picture is favored over processing the distractor by 'blocking' the latter out of the speech production system. This is done by an attentional modulation of the system when it detects both the picture and the distractor. Thus, distractor blocking is seen as an early process that is needed to distinguish between correct and incorrect input information. Furthermore, the speed with which a distractor can be 'blocked' depends on the speed of the availability of the distractor information. As high-frequency words are perceptually available earlier than low-frequency words (e.g., McClelland \& Rumelhart, 1981) their information can be blocked earlier on, making the discrimination between relevant and irrelevant information easier. Consequently, naming latencies will be faster for pictures accompanied by high-frequency distractors. Indeed, computer simulations reported in Roelofs (2005) showed that WEAVER++ can simulate the distractor frequency effect.

In contrast, the response-exclusion hypothesis (e.g., Finkbeiner \& Caramazza, 2006; Mahon et al., 2007) places the effect at a later level. The basic premise is that some of the effects found in the PWI paradigm are taskspecific, not necessarily reflecting lexical selection. The response-exclusion hypothesis makes two assumptions. The first assumption is based on the observation that in spoken language production, only one response at a time can be produced. This leads to the conclusion that word production must involve a single-channel output buffer. The second assumption states that in a PWI task, participants will unavoidably formulate a covert verbal response to the distractor. This response is available before the response to the picture, because words have a privileged relationship to the articulators (e.g., Roelofs, 2003; cf., McLeod \& Posner, 1984; Glaser \& Glaser, 1989). This assumption is supported by the absence of semantic interference when both target and distractor are pictures or when both are words (e.g., Glaser \& Glaser, 1989). In the first case, no response to the distractor is formed, while the second observation can be explained by assuming that the distractor word will be overwritten upon presentation of the target word. Thus, on this account, distractor words will always elicit a covert verbal response. Because of the privileged relationship of words with the articulators, any response that is already in the buffer upon the presentation of the distractor will be overwritten. Therefore, in the PWI paradigm, the response to the distractor needs to be purged from this buffer before a response to the picture can be given.

Because of this need to purge the distractor, the latency of the response to the picture depends on two factors: how fast the response to the distractor enters the buffer, and how fast it is removed. The distractor frequency effect is accounted for by the first factor. Responses to low-frequency words are assumed to be available later due to, for example, the higher resting activation of high-frequency words (e.g., Dell, 1986). As a result, the response to low-frequency distractors is also excluded later and will interfere more, explaining the distractor frequency effect. Therefore, the effect should vary according to whether or not the response to the distractor is formed and/or is in the response buffer. If no response enters the buffer, there will be no operations involving the response buffer. Consequentially, naming latencies should reflect genuine lexical selection processes. Put differently, the distractor frequency effect has a post-lexical locus.

Summarizing, both models assume a form of distractor blocking. In WEAVER++, this is implemented as early attentional modulation designed to allow a differentiation between relevant and irrelevant information. In the response exclusion hypothesis, distractors are removed from a response buffer in order to make room for the response to the picture.

Some studies provides tentative evidence for a late locus of the distractor frequency effect. For example, Miozzo and Caramazza (2003, experiment 4) presented pictures with two types of distractors: those that were and those that were not previously read aloud. They reasoned that if the effect of high-frequency words is attributable to the fact that they were repeatedly selected for production, distractor words that were read aloud before should interfere less. In line with the hypothesis, these distractors indeed led to shorter picture naming latencies. However, this result can also be explained by WEAVER++, by assuming that repeated production leads to faster blocking. Dhooge and Hartsuiker (2010) assumed that, if the distractor frequency effect is indeed due to lowfrequency distractors being excluded slower than highfrequency distractors, the effect should disappear when no response is formed. They presented pictures with their distractors either visible or masked (cf. Finkbeiner \& Caramazza, 2006). The reasoning is that masking should prevent the formulation of a response to the distractor in the buffer (cf. Finkbeiner \& Caramazza, 2008), eliminating the need for late, response-exclusion processes. There was a significant distractor frequency effect under visible conditions, but not under masked conditions. Under masked conditions, the presence of semantic facilitation showed that the absence of the distractor frequency effect was not due to not processing the distractor. Furthermore, it indicated that distractors in the masked condition were processed at least up to the level of semantics. The absence of the distractor frequency effect suggests that the effect has its locus at a later level. Dhooge and Hartsuiker 
(2010) also tested the hypothesis in a different way. They presented low- or high-frequency distractors at various time points before the picture. According to the response exclusion hypothesis, the more time there is between presentation of distractor and picture, the smaller the distractor frequency effect should be. In line with predictions, the distractor frequency effect was only significant when picture and distractor were presented together and when the distractor was presented $100 \mathrm{~ms}$ before the picture. When the distractor was presented $200 \mathrm{~ms}$ or $300 \mathrm{~ms}$ before the picture the effect disappeared. However, WEAVER++ could also account for these data when it is assumed that $100 \mathrm{~ms}$ is not enough to block the distractor out to a sufficient extent.

Another and more direct way to distinguish between the early and late locus is to see whether the distractor frequency effect is present when responses are delayed and lexical selection has already finished. To this purpose, we employed a paradigm introduced by Janssen Schirm, Mahon, and Caramazza (2008), in which participants see pictures with superimposed distractors in an immediate and delayed naming condition. In the immediate naming condition, picture and distractor are presented simultaneously. In the delayed naming condition, the picture is presented $1000 \mathrm{~ms}$ before the distractor. Depending on the color of the distractor, either the distractor or the picture has to be named. The crucial condition is the delayed naming condition. In this condition, participants are asked to always prepare the picture name, despite the fact that they sometimes have to name the distractor. This ensures that upon distractor presentation, lexical selection of the picture name has already been completed, which predicts no effect of picture frequency. According to the response exclusion hypothesis, presenting the distractor will always lead to the formulation of a response to the distractor. This response will enter the response buffer, overwriting the response to the picture that is presumably located in the buffer. If the task is then to name the picture, the response to the distractor needs to be purged from the buffer, leading to the distractor frequency effect. However, according to WEAVER++, the response is already available and ready for production. As it is clear which response is correct, there is no need for selection anymore, and consequently, for distractor blocking. Thus, there should not be a distractor frequency effect. The immediate naming condition is the standard condition. In this condition, participants cannot have retrieved the picture name upon presentation of the distractor as they are presented simultaneously. Therefore, both WEAVER++ and the response exclusion hypothesis predict an effect of both picture frequency and distractor frequency.

\section{Method}

Participants Fifty-three native speakers of Dutch took part in the experiment and received $€ 8$ for their participation.

Stimuli We selected 40 pictures from the Severens, Van Lommel, Ratinckx, and Hartsuiker (2005) database. Twenty had low-frequency names and 20 had high-frequency names based on the CELEX lexical database (Baayen, Piepenbrock, \& Van Rijn, 1993). Combining the pictures resulted in 20 pairs. For each picture pair, a low-frequency and high-frequency distractor was selected. Thus, for each combination of picture frequency and distractor frequency there were 20 items, resulting in a total of 80 experimental items (see Appendix). Pictures and words were paired such that there was no phonological or semantic relationship between them. Repeated measures ANOVAs showed no significant differences in the number of letters (perfect match), number of syllables, number of phonemes, number of neighbors, and word bigram frequency (all $p>0.38$; see Table 1).

Low- and high-frequency items differed significantly in frequency, $\mathrm{F}(1,19)=569.47, p<0.001$, but there was no effect of item type (picture or word) and no significant interaction (both $\mathrm{F}<1$ ). Paired samples $t$ tests showed a

Table 1 Features of low-frequency (LF) and high-frequency (HF) pictures and distractors (standard deviations between parentheses)

\begin{tabular}{lllll}
\hline & LF picture & HF picture & LF word & HF word \\
\hline Log frequency & $0.62(0.35)$ & $2.19(0.27)$ & $0.61(0.32)$ & $2.24(0.26)$ \\
Letters & $4.60(1.14)$ & $4.60(1.14)$ & $4.60(1.14)$ & $4.60(1.14)$ \\
Syllables & $1.25(0.44)$ & $1.25(0.55)$ & $1.25(0.44)$ & $1.20(0.41)$ \\
Phonemes & $4.05(1.00)$ & $3.85(1.04)$ & $4.00(1.21)$ & $4.00(0.79)$ \\
Neighbors & $7.95(5.97)$ & $8.45(6.50)$ & $7.45(6.14)$ & $6.55(4.62)$ \\
Bigram frequency & $36056.8(23356.48)$ & $33207.49(24662.49)$ & $28574.7(21283.45)$ & $34197.85(20953.36)$ \\
\hline
\end{tabular}


significant difference in frequency between low- and highfrequency pictures, $\mathrm{t}(19)=-13.67, p<0.001$; and lowand high-frequency words, $\mathrm{t}(19)=-17.20, p<0.001$. Twelve pictures and 12 words that did not appear in the experiment proper were selected for the practice phase.

A plus sign ('+') served as fixation point. All pictures were 300 x 300 pixels large and presented centrally on screen. Distractor words were presented in a Times New Roman font 26 points centrally at fixation. Oral responses were collected through a voice key connected to the parallel port.

Procedure First, the participants were familiarized with the names of all pictures. Each trial started with the presentation of the fixation cross for $700 \mathrm{~ms}$ followed by the picture. After 1,000 ms, the picture name appeared, forming a cue to name the picture. The next trial was initiated $1,000 \mathrm{~ms}$ after the experimenter had typed in whether the response was correct or not.

Second, we presented two blocks of trials, one block of each condition. The order of conditions was counterbalanced across participants. In the delayed naming condition, each trial started with the presentation of a fixation cross for $700 \mathrm{~ms}$, which was replaced by a picture. The distractor word appeared $1,000 \mathrm{~ms}$ later and formed a cue to give a response. The picture and distractor stayed on screen for $500 \mathrm{~ms}$, or less when the participant had already responded. Subsequently, the experimenter typed in whether the response was correct or not. The next trial was initiated after $500 \mathrm{~ms}$. In the immediate naming condition, the trial sequence was identical but after the fixation cross, the picture and distractor appeared simultaneously. In both conditions, the task depended on the color of the distractors. If the distractor was dark blue, the task was to name the distractor. However, if the distractor was black, the task was to name the picture. Each block started with a practice phase of 24 trials, which followed the trial sequence of the corresponding block. Each experimental block consisted of 160 trials. Each picture appeared with its low- and with its high-frequency distractor twice: once in the condition where the picture had to be named (experimental trials) and once in the condition where the distractor had to be named (filler trials). This resulted in 320 experimental trials. Trials were randomized with the constraints that (1) all pictures were presented once before they were repeated, (2) no item from the same condition (distractor frequency, picture frequency and distractor versus picture naming) appeared on more than three consecutive trials. Whether a picture appeared first with its low- or high-frequency distractor and in the picture or distractor naming condition, was counterbalanced across participants with a Latin Square design with four lists. Between the different blocks, and halfway through the experimental blocks, participants could take a self-paced break. Importantly, in the delayed naming condition, participants were instructed to always prepare the picture name upon its onset, even though they might have to name the distractor.

\section{Results}

We decided to score responses as errors when they included (a) voice key malfunctioning and verbal disfluencies and (b) incorrect naming of the picture; but no naming errors were made. Additionally, all responses exceeding the participant's mean by three standard deviations and naming latencies below $300 \mathrm{~ms}$ were discarded from the analyses. Errors and outliers accounted for $0.90 \%$ and $1.76 \%$ of the data and were not further analyzed.

We performed repeated measures ANOVAs (see Table 2), one with participants as a random effect and one with items as a random effect on responses latencies. Three variables were considered: picture frequency (2; low-frequency vs. high-frequency, within-subjects and between-items), distractor frequency (2; low-frequency vs. high-frequency, within-subjects and within items) and condition (2, delayed vs. immediate, within-subjects and within-items).

Omnibus analysis Naming latencies were faster in the delayed than in the immediate naming condition, $\mathrm{F}_{1}(1$, $52)=383.91, p<0.001, \mathrm{~F}_{2}(1,38)=1,608.07, p<0.001$,

Table 2 Average naming latencies (in ms, standard errors between parentheses) for the delayed and immediate naming condition

\begin{tabular}{|c|c|c|c|c|c|c|}
\hline & \multicolumn{3}{|c|}{ Delayed naming condition } & \multicolumn{3}{|c|}{ Immediate naming condition } \\
\hline & LF distractor & HF distractor & difference & LF distractor & HF distractor & difference \\
\hline LF picture & $626(16.54)$ & $613(15.48)$ & $13(4.73)$ & $902(17.09)$ & $869(16.35)$ & $33(5.51)$ \\
\hline HF picture & $623(15.86)$ & $611(16.45)$ & $12(6.01)$ & $844(14.96)$ & $829(13.93)$ & $15(6.96)$ \\
\hline difference & $3(6.04)$ & $2(5.92)$ & & $58(7.02)$ & $40(7.04)$ & \\
\hline
\end{tabular}


they were faster for high-frequency than low-frequency pictures $\mathrm{F}_{1}(1,52)=43.41, p<0.001, \mathrm{~F}_{2}(1,38)=7.02$, $p<0.05$, and faster in the context of high-frequency than low-frequency words, $\mathrm{F}_{1}(1,52)=27.13, p<0.001, \mathrm{~F}_{2}(1$, $38)=19.23, p<0.001$. There was a significant interaction between condition and picture frequency, $\mathrm{F}_{1}(1,52)=$ 41.76, $p<0.001, \mathrm{~F}_{2}(1,38)=15.22, p<0.001$. There was a significant picture frequency effect in the immediate naming condition, $\mathrm{t}_{1}(52)=8.69, p<0.001, \mathrm{t}_{2}(38)=3.59$, $p<0.001$, but not in the delayed naming condition, both $t<1$. The interactions between condition and distractor frequency, $\mathrm{F}_{1}(1,52)=4.05, p=0.05, \mathrm{~F}_{2}(1,38)=1.96$, $p=0.17$, picture frequency and distractor frequency, $\mathrm{F}_{1}(1$, $52)=3.31, p=0.08, \mathrm{~F}_{2}(1,38)=1.14, p=0.29$, and the three-way interaction $\mathrm{F}_{1}(1,52)=3.63, p=0.09, \mathrm{~F}_{2}$ $(1,38)=1.14, p=0.29$, did not reach conventional levels of significance. To characterize this pattern of results more in detail, we analyzed the results of both naming conditions separately.

Immediate naming Naming latencies were shorter for high-frequency than low-frequency pictures, $\mathrm{F}_{1}(1,52)=$ $74.70, p<0.001, \mathrm{~F}_{2}(1,38)=12.96, p<0.01$. The effect of distractor frequency was also significant, $\mathrm{F}_{1}(1,52)=$ $22.05, p<0.001, \mathrm{~F}_{2}(1,38)=10.87, p<0.01$. The interaction between these two variables was significant by subjects, $\mathrm{F}_{1}(1,52)=5.30, p<0.05$, but not by items, $\mathrm{F}_{2}(1,38)=1.54, p=0.22$. Paired samples $t$ tests showed a significant effect of distractor frequency with lowfrequency pictures, $\mathrm{t}_{1}(52)=5.27, p<0.001, \mathrm{t}_{2}(19)=$ 2.98, $p<0.01$. With high-frequency pictures, the distractor frequency effect was significant only by subjects, $\mathrm{t}_{1}(52)=2.09, p<0.05, \mathrm{t}_{2}(19)=1.59, p=0.13$.

Delayed naming There was no effect of picture frequency, both $\mathrm{F}<1$. Importantly, naming latencies were shorter with a high compared to a low-frequency distractor, indicating a distractor frequency effect, $\mathrm{F}_{1}(1,52)=8.08, p<0.01$, $\mathrm{F}_{2}(1,38)=7.48, p<0.01$. The interaction was not significant, both $\mathrm{F}<1$.

\section{Discussion}

The main result from this study is the observation of the distractor frequency effect in the delayed naming condition, while there was clearly no effect of picture frequency in that condition. The latter finding can be interpreted as indicating that the lexical representation of the picture name had already been retrieved when the distractor was presented (see also Janssen et al., 2008). Thus, even when lexical selection had already taken place, the distractor still influenced naming latencies. These results support a late, post-lexical origin of the DFE. According to the response exclusion hypothesis, when the picture is presented in the delayed naming condition, the response to the picture should enter the response buffer. However, the fact that there is a DFE in this condition indicates that the response to the distractor displaces the response to the picture in the buffer. Therefore, it needs to be removed, leading to the DFE.

Unexpectedly, picture frequency and distractor frequency seemed to interact in the immediate naming condition (but note that this interaction was not significant by-items). If genuine, this interaction might result from an interplay between the processes of response exclusion and picture name preparation which, according to the response exclusion hypothesis, overlap in time. Assuming that both processes draw on a limited amount of computational resources (see, e.g., Ferreira \& Pashler, 2002 , for the proposal that lexical access is constrained by limited capacity), and assuming that low-frequency items (pictures or words) make a larger demand on this capacity than high-frequency items do (e.g., Goldinger, Azuma, Abramson, \& Jain, 1997), then there might be an extra processing cost when both the picture and distractor are of low frequency. In other words, a low-frequency distractor will always be excluded later than a highfrequency distractor, but the exclusion process will experience an even greater delay in the condition with low-frequency pictures, because now both the exclusion and preparation processes make a relatively large demand on the resources, and this combined demand might exceed capacity. In the delayed naming condition, processing of the picture response will not compete for resources with processing of the distractor, as the picture response is already prepared. Therefore, there should not be an interaction.

Finally, we note that the response exclusion hypothesis still needs to be further specified. Most importantly, the nature of the control mechanism operating over the response buffer is not made explicit. Dhooge and Hartsuiker (2010) suggested that one possibility could be that the checking of the response is done by the verbal self-monitor (e.g., Hartsuiker \& Kolk, 2001). The normal function of the monitor is to check external speech for speech errors, inappropriate words, etc. The verbal self monitor could also check an internal speech plan for correctness, and as such prevent errors in the PWI paradigm (for a more detailed proposal of the role of self-monitoring in the PWI paradigm, see Dhooge \& Hartsuiker, 2010).

In conclusion, our data suggest that the locus of the distractor frequency effect can be situated at a post-lexical level, which is in line with the response exclusion hypothesis. 
Author note This research was supported by Fund for Scientific

Research Flanders Grand FWO08/ASP/070 to Elisah Dhooge.

\section{Appendix}

Table 3 Materials: low-frequency (LF) and high-frequency (HF) pictures and distractors (English translations between parentheses)

\begin{tabular}{|c|c|c|c|}
\hline LF picture & HF picture & LF word & HF word \\
\hline $\operatorname{Vos}(f o x)$ & Steen (stone) & Rits (zipper) & Koning (king) \\
\hline Kan (jug) & Deur (door) & Sjaal (scarf) & Vogel (bird) \\
\hline Harp (harp) & Oog (eye) & Ton (barrel) & Stad (city) \\
\hline Peer (pear) & Telefoon (telephone) & Krab (crab) & Bed (bed) \\
\hline Zaag (saw) & Muur (wall) & Uil (owl) & Blad (leaf) \\
\hline Kruk (stool) & Spoor (track) & IJsje (ice cream) & Blik (can) \\
\hline Stop (plug) & Kat (cat) & Drumstel (drum) & Arm (arm) \\
\hline Kers (cherry) & Boek (book) & Slak (snail) & Traan (tear) \\
\hline Hoef (hoof) & Tafel (table) & Stok (cane) & Bloem (flower) \\
\hline Tang (pliers) & Bureau (desk) & Hooi (hay) & Schouder (shoulder) \\
\hline Hert (deer) & Glas (glass) & Spuit (syringe) & Brief (letter) \\
\hline Broek (pants) & Fles (bottle) & Mier (ant) & Trap (stairs) \\
\hline Gewei (antlers) & Vinger (finger) & Slee (sledge) & Kerk (church) \\
\hline Anker (anchor) & Brood (bread) & Gieter (watering can) & Raam (window) \\
\hline Zebra (zebra) & Stoel (chair) & Banaan (banana) & Hand (hand) \\
\hline Strik (bow) & Paard (horse) & Mais (corn) & Geest (ghost) \\
\hline Taart (pie) & Neus (nose) & Spin (spider) & Beeld (statue) \\
\hline Schaar (scissors) & Hond (dog) & Clown (clown) & Wijn (wine) \\
\hline Ballon (balloon) & Hart (heart) & Robot (robot) & Vuur (fire) \\
\hline Trechter (funnel) & Been (leg) & Zwaan (swan) & Papier (paper) \\
\hline
\end{tabular}

\section{References}

Baayen, R. H., Piepenbrock, R., \& Van Rijn, H. (1993). The CELEX lexical database. Philadelphia: CD-ROM.

Burt, J. S. (2002). Why do non-color words interfere with color naming? Journal of Experimental Psychology: Human Perception and Performance, 28(A), 1019-1038. doi:10.1037//0096-1523.28.5.1019

Catling, J. C., Dent, K., Johnston, R. A., \& Balding, R. (2010). Age of acquisition, word frequency, and picture-word interference. The Quarterly Journal of Experimental Psychology, 63, 1304-1317. doi: $10.1080 / 17470210903380830$

Dell, G. S. (1986). A spreading-activation theory of retrieval in sentence production. Psychological Review, 93, 283-321.

Dhooge, E., \& Hartsuiker, R. J. (2010). The distractor frequency effect in picture-word interference: Evidence for response exclusion. Journal of Experimental Psychology. Learning, Memory, and Cognition, 36, 878-891. doi:10.1037/a0019128

Ferreira, V. S., \& Pashler, H. (2002). Central bottleneck influences on the processing stages of word production. Journal of Experimental Psychology. Learning, Memory, and Cognition, 28, 11871199. doi:10.1037//0278-7393.28.6.1187
Finkbeiner, M., \& Caramazza, A. (2006). Now you see it, now you don't: On turning semantic interference into facilitation in a Stroop-like task. Cortex, 42, 790-796.

Finkbeiner, M., \& Caramazza, A. (2008). Modulating the masked congruence priming effect with the hands and the mouth. Journal of Experimental Psychology: Human Perception and Performance, 34, 894-918. doi:10.1037/ 0096-1523.34.4.894

Glaser, W. R., \& Glaser, M. O. (1989). Context effects on Stroop like word and picture processing. Journal of Experimental Psychology: General, 118, 13-42.

Goldinger, S. D., Azuma, T., Abramson, M., \& Jain, P. (1997). Open wide and say "Blah!": Attentional dynamics of delayed naming. Journal of Memory and Language, 37, 190-216.

Hartsuiker, R. J., \& Kolk, H. H. K. (2001). Error monitoring in speech production: A computational test of the perceptual loop theory. Cognitive Psychology, 42, 113-157.

Janssen, N., Schirm, W., Mahon, B. Z., \& Caramazza, A. (2008). Semantic interference in a delayed naming task: Evidence for the response exclusion hypothesis. Journal of Experimental Psychology. Learning, Memory, and Cognition, 34, 249-256. doi:10.1037/0278-7393.34.1.249 
Levelt, W. M. J., Roelofs, A., \& Meyer, A. S. (1999). A theory of lexical access in speech production. The Behavioral and Brain Sciences, 22, 1-75.

MacLeod, C. M. (1991). Half a century of research on the Stroop effect: An integrative review. Psychological Bulletin, 109, 163203.

Mahon, B. Z., Costa, A., Peterson, R., Vargas, K. A., \& Caramazza, A. (2007). Lexical selection is not by competition: A reinterpretation of semantic interference and facilitation effects in the picture-word interference paradigm. Journal of Experimental Psychology. Learning, Memory, and Cognition, 33, 503-533. doi:10.1037/0278-7393.33.3.503

McClelland, J. L., \& Rumelhart, D. E. (1981). An interactive activation model of context effects. Psychological Review, 88, 375-407.

McLeod, P., \& Posner, M. I. (1984). Privileged loops from percept to act. In H. Bouma \& D. G. Bouwhuis (Eds.), Attention and performance $(X)$ (pp. 55-66). Hillsdale: Erlbaum.
Miozzo, M., \& Caramazza, A. (2003). When more is less: A counterintuitive effect of distractor frequency in picture-word interference paradigm. Journal of Experimental Psychology: General, 132, 228-252. doi:10.1037/0096-3445.132.2.228

Roelofs, A. (2003). Goal-referenced selection of verbal action: Modeling attentional control in the Stroop task. Psychological Review, 10, 88-125. doi:10.1016/j.jml.2006.10.001

Roelofs, A. (2005). From Popper to Lakatos: A case for cumulative computational modeling. In A. Cutler (Ed.), Twenty-first century psycholinguistics: Four cornerstones (pp. 313-330). Hillsdale: LEA.

Severens, E., Van Lommel, S., Ratinckx, E., \& Hartsuiker, R. J. (2005). Timed picture naming norms for 590 pictures in Dutch. Acta Psychologica, 119, 159-187. doi:10.1016/j. actpsy.2005.01.002

Schriefers, H., Meyer, A. S., \& Levelt, W. J. M. (1990). Exploring the time course of lexical access in language production-picture word interference studies. Journal of Memory and Language, 29, 86-102. 